\title{
PARALISIA FACIAL PERIFÉRICA DE BELL: ATUALIZAÇÃO DO TRATAMENTO
}

\author{
Igor Henrique Balandino Silva \\ Universidade Severino Sombra, Centro de Ciências da Saúde, Curso de Medicina, \\ Interno do curso de medicina, igorvga@yahoo.com.br
}

\section{Tiago de Souza Lopes} Universidade Severino Sombra, Centro de Ciências da Saúde, Curso de Medicina,
Interno do curso de medicina, tiagoiguatulahotmail.com

\section{Elisa Nocchi de Mello Motta}

Universidade Severino Sombra, Centro de Ciências da Saúde, Curso de Medicina, Interno do curso de medicina, dra_elisamotta@hotmail.com

\section{Juliane Lemos Araújo Deveras}

Universidade Severino Sombra, Centro de Ciências da Saúde, Curso de Medicina,
Interno do curso de medicina, julideverasahotmail.com Paula Corrêa Côrtes

Universidade Severino Sombra, Centro de Ciências da Saúde, Curso de Medicina, Interno do curso de medicina, paula_britog@hotmail.com

\section{Carla Carvalho Silva Marques}

Universidade Severino Sombra, Centro de Ciências da Saúde, Curso de Medicina, Interno do curso de medicina, carlinhagbi@hotmail.com

\section{Clara Régia de Sousa Rosa}

Universidade Severino Sombra, Centro de Ciências da Saúde, Curso de Medicina, Interno do curso de medicina, clararegia@yahoo.com.br

\section{Vinicius Florentino da Silva}

Universidade Severino Sombra, Centro de Ciências da Saúde, Curso de Medicina, Interno do curso de medicina, vini_florentino@hotmail.com

\section{Ana Beatriz Calmon Nogueira da Gama Pereira}

\footnotetext{
Universidade Severino Sombra, Centro de Ciências da Saúde, Curso de Medicina, Professora Assistente das Disciplinas de Enfermidades Prevalentes em Neurologia. Médica do Serviço de Neurologia do Hospital Universitário Sul Fluminense, anacalmon@uol.com. br
}

Resumo. A paralisia facial periférica (PFP) caracteriza-se pela interrupção, temporária ou não, dos movimentos da musculatura facial, sendo a Paralisia de Bell (PB) a mais prevalente. Afeta ambos os sexos; as hemifaces são acometidas com a mesma frequência; a incidência é maior após 70 anos e menor em indivíduos com menos de 10 anos. A PFP pode estar associada à otite média aguda e o vírus Herpes simplex 1 (HSV-1). Objetivo: Analisar e revisar retrospectivamente os principais tópicos relacionados à $P B$, enfatizando a atualização do tratamento. Metodologia: Levantamento bibliográfico retrospectivo publicado durante o período de 2005 a 2010. Os artigos foram analisados e apresentados de forma descritiva sob o aspecto medicamentoso, fisioterápico $e$ fonoaudiológico do tratamento. Conclusão: Ocorre benefício terapêutico com o uso de prednisolona juntamente com o valaciclovir no momento do diagnóstico da paralisia. 
Ainda assim o tratamento da PB necessita de mais estudos para uma melhor abordagem terapêutica.

Palavras-Chave: Paralisia facial periférica, paralisia de Bell, paralisia facial idiopática, tratamento.

\title{
BELL of Peripheral Facial Paralysis: Treatment Update
}

\begin{abstract}
The facial palsy $(P F P)$ is characterized by the interruption, temporary or not, the movement of facial muscles, and Bell's Palsy $(C P)$ the most prevalent. It affects both sexes, the hemifaces are affected with equal frequency, and the incidence is higher after 70 years and lowest in individuals younger than 10 years. The PFP may be associated with acute otitis media and Herpes simplex virus 1 (HSV-1). Objective: To analyze retrospectively reviewed and the main topics related to $C P$, emphasizing the update treatment. Methodology: A literature published during the retrospective period from 2005 to 2010. The articles were analyzed and presented in a descriptive way in the aspect medication, physical therapy and speech therapy treatment. Conclusion: There was therapeutic benefit with the use of prednisone with valacyclovir at diagnosis of paralysis. $Y e t$ the treatment of $P B$ requires further studies to better therapeutic approach.
\end{abstract}

Keywords: Facial paralysis, Bell's palsy, idiopathic facial paralysis, treatment.

\section{Introdução}

A paralisia facial periférica caracteriza-se pela interrupção, temporária ou não, dos movimentos da musculatura facial. Pode acompanhar-se de alterações na secreção salivar e na secreção lacrimal, assim como nas sensibilidades facial e auditiva. Dentre as causas encontram-se a idiopática ou de Bell, traumática, tumoral, infecciosa ou decorrente de outras causas. A paralisia facial periférica gera prejuízo nas funções orais como fala, mastigação, sucção, deglutição e preensão labial, além de comprometimentos de ordem estética com repercussão emocional significativa (Tessitore, Paschoal, e Pfeilsticker, 2009).

A paralisia facial periférica foi descrita por Sir Charles Bell em 1821. Inicialmente, todos os quadros de paralisia do nervo facial passaram a ser chamados de Paralisia de Bell. Entretanto, com a descoberta de causas da doença, apenas os quadros idiopáticos mantiveram esta denominação. A Paralisia de Bell corresponde de $60 \%$ a $75 \%$ de todas as causas de paralisia facial. Sua incidência foi estimada em 13 a 34 casos em cada 100.000 pessoas. Embora seja o tipo mais frequente de paralisia facial periférica, a causa da Paralisia de Bell ainda é objeto de inúmeras pesquisas, teorias e questionamentos. Afeta ambos os sexos. As hemifaces são acometidas com a mesma frequência e a incidência é maior em pessoas com mais de 70 anos (53 por 100.000) e menor em indivíduos com menos de 10 anos (4 por 100.000) (Falavigna, Teles, Giustina e Kleber, 2008).

Atualmente inúmeros estudos buscam correlações da paralisia facial periférica com a otite média aguda e o vírus Herpes simplex 1 (HSV-1). Yonamine et al. (como citado em Santos, Filho, Viana, Almeida e Lazarini, 2010) relata que a paralisia facial periférica associada à otite media aguda não é muito frequente nos dias atuais devido a introdução dos antibióticos. Atingem de $1 \%$ a $4 \%$ dos casos de paralisias faciais das diversas casuísticas. Lazarini et al. (como citado em Santos, et al., 2010) refere-se a presença do HSV-1 na saliva de pacientes portadores de paralisia facial periférica de Bell indicando que a reativação viral pode ser a etiologia desta doença.

O tratamento da paralisia de Bell requer abordagem médica, fisioterapêutica e fonoaudiológica. Pode ser medicamentoso apenas ou associado à terapia de reabilitação ou ainda medicamentoso e cirúrgico seguido da reabilitação orofacial. 


\section{Paralisia Facial Periférica de Bell: Atualização do Tratamento}

Igor Henrique Balandino Silva, Tiago de Souza Lopes, Elisa Nocchi de Mello Motta, Juliane Lemos Araújo Deveras, Paula Corrêa Côrtes, Carla Carvalho Silva Marques, Clara Régia de Sousa Rosa, Vinicius Florentino da Silva e Ana Beatriz Calmon Nogueira da Gama Pereira

\section{Objetivos}

O objetivo deste trabalho é analisar e revisar retrospectivamente os principais tópicos relacionados à paralisia de Bell, enfatizando a atualização do tratamento. $\mathrm{O}$ enfoque foi à abordagem terapêutica quanto à associação de corticosteróides e antirretrovirais assim como sua eficácia associada a outros métodos terapêuticos não medicamentosos, afim de um melhor prognóstico aos pacientes com paralisia facial periférica de Bell.

\section{Metodologia}

Foi realizado um levantamento bibliográfico retrospectivo, utilizando-se as palavras-chave "paralisia de Bell, palsy Bells, parálisis de Bell" nos indexadores MEDLINE (Literatura Internacional em Ciências da Saúde), LILACS (Literatura Latinoamericana em Ciências da Saúde), COCHRANE, publicados durante o período de 2005 a 2010, em língua portuguesa, inglesa e espanhola. Foram encontrados 19 artigos correspondentes a pesquisa, na qual foram selecionados 12 artigos referentes à atualização no manejo e tratamento da paralisia facial de Bell.

Os artigos foram analisados e apresentados de forma descritiva sob o aspecto medicamentoso, fisioterápico e fonoaudiológico do tratamento.

\section{Revisão de Literatura}

Ocorrem relatos de paralisia facial periférica nas artes plásticas no Egito antigo, Grécia e Roma, Idade Média, Renascimento e também dos últimos quatro séculos. Pensa-se que a história da paralisia facial periférica acompanha a história da própria espécie humana (Resende, \& Weber, 2008).

A paralisia facial periférica de acordo com Martins e Lima (1999), e Almeida (1992) (como citado em Soares, Silva e Bertolini, 2002) afeta homens e mulheres proporcionalmente em qualquer faixa etária, e é observada uma maior incidência de Paralisia Facial Periférica em indivíduos com média de 35,5 anos.

Estima-se que a incidência da paralisia de Bell seria de 20-30 por 100 mil habitantes. É raro antes dos 10 anos de idade e sua incidência é bimodal, com picos na terceira e oitava décadas de vida, dependendo da distribuição etária da população (Valença, Valença e Lima, 2001).

A etiologia da paralisia facial periférica é variável e inclui causas neoplásicas e metabólicas, afecções inflamatórias da orelha média, herpes zoster, mas as mais comuns são de ordem idiopática ou de Bell, seguida da traumática (fragmentos de arma de fogo, traumatismos cranianos, ferimentos cortantes da face e lesões iatrogênicas). Desde que se descreveu pela primeira vez em 1830, tem-se falado em diversas teorias para explicar a etiopatogenia da paralisia facial periférica de Bell; as mais recentes incluem a hipótese da isquemia vascular, imunológica e de compressão, e nos últimos anos tem surgido a hipótese virótica (por Herpes simplex), com diversas investigações que a apoiam (Rosa, Moreira, Araújo, Júnior e Motta, 2010).

A hipótese da isquemia vascular leva em conta a isquemia primária e a isquemia secundária. A primeira delas postula que a paralisia aparecia como uma consequência de uma disfunção do sistema nervoso autônomo, que ocasionava espasmo e trombose nos vasos nutricionais do nervo. A segunda teoria especulou sobre a possibilidade de que o edema fosse consequência de um processo inflamatório, viral ou imunológico, que produzisse uma alteração na microcirculação do nervo e uma diminuição da condutividade neural. A hipótese imunológica propõe que ocorre uma reação imunológica de hipersensibilidade, localizada no nervo facial. Os fatores etiológicos desencadeantes poderiam ser estímulos mecânicos, químicos, traumáticos e fundamentalmente infecções virais, que iniciariam a sequência de alterações imunológicas (Diego, Prim e Gavilán, 2001). Em 1972, Fisch e

Revista de Saúde, Vassouras, v. 3, n. 2, p. 40-48, jul./dez., 2012 


\section{Paralisia Facial Periférica de Bell: Atualização do Tratamento}

Igor Henrique Balandino Silva, Tiago de Souza Lopes, Elisa Nocchi de Mello Motta, Juliane Lemos Araújo Deveras, Paula Corrêa Côrtes, Carla Carvalho Silva Marques, Clara Régia de Sousa Rosa, Vinicius Florentino da Silva e Ana Beatriz Calmon Nogueira da Gama Pereira

Esslen (citado por Diego, et al., 2001) expuseram uma hipótese sobre a etiopatogenia da Paralisia Facial de Bell, baseada nos achados durante as descompressões do nervo facial em seu trajeto intratemporal. Observaram que, a maior parte dos enfermos - 11 de 12 pacientes - as alterações se encontraram na porção proximal do gânglio geniculado, entre este e o conduto auditivo interno.

McCormick (1972) (como citado em Falavigna et al., 2008) postulou que a reativação do vírus Herpes simplex (VHS) no gânglio geniculado do nervo facial poderia estar associada com a paralisia de Bell. Desde essa publicação, inúmeros autores têm demonstrado associação entre VHS e paralisia de Bell.

O estudo realizado por Murakami et al. (1996) (como citado em Falavigna et al., 2008) detectou a presença do VHS no líquido endoneural em 11 de 14 pacientes com paralisia de Bell. Além disso, alguns autores observaram a associação de infecção oral recente pelo VHS e desenvolvimento de paralisia facial periférica unilateral e bilateral.

Muitos pacientes com paralisa de Bell associam a paralisia com a exposição brusca ao frio. Alguns estudos epidemiológicos foram conduzidos com propósito de avaliar os efeitos climáticos no desenvolvimento da paralisia de Bell. (Falavigna et al., 2008).

Campbelle e Brundage (2002, citado por Falavigna et al., 2008) estudaram os efeitos do clima, da latitude e das estações do ano na incidência de paralisia de Bell em militares norteamericanos. $\mathrm{Na}$ análise de 1181 casos, os autores concluíram que a residência em climas áridos e a exposição ao frio são fatores de risco independentes para o desenvolvimento da paralisia de Bell. A exposição ao frio e ao ar seco, como durante os meses de inverno em regiões de clima árido, poderia traumatizar a mucosa da nasofaringe o que poderia causar a reativação da infecção pelo VHS.

A paralisia facial periférica caracteriza-se por uma lesão do nervo facial em todo o seu trajeto ou parte dele. Esta lesão pode ser causada por fatores traumáticos, infecciosos, neoplásicos, congênitos, metabólicos e sistêmicos. Quando o quadro clínico de paralisia facial periférica não está associada a nenhum destes fatores, considera-se o diagnóstico de paralisia de Bell, também denominada frigore ou idiopática, sendo assim um diagnóstico de exclusão. A paralisia facial idiopática corresponde à maioria dos casos de paralisia facial periférica aguda (Goulart, Vasconcelos, Souza e Pontes 2002).

O principal sintoma da paralisia de Bell é a paresia facial súbita. Aproximadamente $50 \%$ dos pacientes queixam-se de dor retroauricular que persiste por alguns dias, em metade desses pacientes a dor aparece dois a três dias antes da paralisia e, nos demais, ela ocorre no momento da sua instalação. A diminuição da sensibilidade gustativa e da produção de lágrimas é observada em $30 \%$ e 5\% dos casos, respectivamente. Durante a recuperação do quadro o paciente pode cursar com o fenômeno de lacrimejamento após um estímulo salivatório, conhecido como síndrome das lágrimas de crocodilo, esse fenômeno está presente em $70 \%$ dos pacientes. Hiperacusia é identificada em aproximadamente $15 \%$ dos pacientes (Falavigna et al., 2008).

Os principais diagnósticos diferenciais da paralisia facial periférica incluem: paralisia facial de origem central, paralisia facial congênita, paralisia de origem traumática, paralisia facial lentamente progressiva, paralisia unilateral recorrente, paralisia facial bilateral simultânea e doenças Infecciosas como herpes zoster, otite média, hanseníase, sífilis e doença de Lyme (Chávez et al., 2004).

O tratamento da paralisia facial periférica continua sendo muito controverso, já que a taxa de recuperação espontânea se aproxima de 70 \% e a evidencia que dispomos a respeito da efetividade dos distintos tratamentos são insuficientes (Brosens e Botargues, 2008).

Revista de Saúde, Vassouras, v. 3, n. 2, p. 40-48, jul./dez., 2012 
$\mathrm{Na}$ paralisia facial periférica a equipe médica deve atuar rapidamente para estabelecer o diagnóstico e o tratamento mais adequado a cada momento e evitar possíveis sequelas motoras faciais (Lazarini, et al., 2006).

\section{Discussão}

O tratamento da paralisia de Bell é polêmico devido à falta de grandes estudos randomizados, controlados e prospectivos. Há indícios de que os esteroides ou agentes antivirais são benéficos, mas também alguns estudos demonstram resultados controversos. Outras medidas incluem proteção para os olhos, a fisioterapia, a acupuntura, a toxina botulínica, ou possivelmente a cirurgia (Teixeira, Prado, Vieira e Soares, 2008).

Os principais objetivos do tratamento incluem a velocidade de recuperação, a fim de evitar as complicações da córnea e outras sequelas, além de inibir a replicação viral. O apoio psicológico também é essencial. Pacientes com paralisia de Bell devem ser encaminhados para um especialista e o tratamento deve ser iniciado o mais rápido possível, podendo ser subdividido em tratamentos imediatos e tratamentos para as sequelas moderadas ou graves (Finsterer, 2008).

\section{Tratamento Fonoaudiológico e Fisioterápico}

Tessitore, Paschoal e Pfeilsticker (2009) realizaram um estudo prospectivo longitudinal de avaliação de um protocolo de reabilitação orofacial utilizado no tratamento da paralisia facial periférica. $\mathrm{O}$ estudo foi objetivado no atendimento fonoaudiológico, utilizando massagens orofaciais, solicitação funcional com inibição do lado sadio e uso de força corporal associada à solicitação funcional. No atendimento fisioterápico solicitou-se elevação e aproximação das sobrancelhas, fechamento do olho, contração nasal, inflar bochechas, apertar lábios, fazer bico, sorrir e extensão máxima dos cantos da boca. A partir daí segue-se com os exercícios miofuncionais. O protocolo de reabilitação das paralisias faciais periféricas avaliado foi eficaz, nesta amostra, na promoção de melhoras do repouso facial, ao garantir aumento marcante do tônus muscular, conforme aferição do ângulo da comissura labial. Este constitui uma alternativa recomendável na reabilitação da Paralisia Facial Periférica.

Teixeira et al. (2008) analisou em sua pesquisa a ação da fisioterapia no tratamento da paralisia facial periférica. A pesquisa identificou 45 artigos potencialmente relevantes, e este estudo serviu para avaliar a eficácia de terapias físicas sobre o resultado da paralisia de Bell. Foram selecionados estudos randomizados ou quasi-randomizados e controlados envolvendo toda a terapia física. Foram incluídos no estudo participantes de qualquer idade com diagnóstico de paralisia de Bell e de todos os graus de severidade. As medidas de desfecho foram analisadas sob os seguintes aspectos: recuperação incompleta seis meses após a randomização, sincinesia motora, lágrimas de crocodilo ou espasmo facial seis meses após o início, a recuperação incompleta após um ano e os efeitos adversos atribuíveis à intervenção. Segundo os autores não há nenhuma evidência de benefício significativo ou prejuízo de quaisquer fisioterapias para paralisia facial idiopática. A possibilidade de que o exercício facial reduz o tempo para recuperar sequelas, é necessário confirmar com boa qualidade de ensaios clínicos randomizados.

Rosa et al (2010) comparou a funcionabilidade da fonoterapia isolada e fonoterapia associada à acupuntura. Foi realizado um estudo prospectivo comparativo, no período de julho a outubro de 2007 de um grupo de 15 pacientes com a paralisia facial periférica. Dentre eles, foi feita uma seleção randomizada de 8 pacientes que foram submetidos à fonoterapia e acupuntura (grupo experimental), enquanto os outros 7 pacientes (grupo controle) foi submetido apenas à fonoterapia. Quanto às medidas faciais investigadas houve melhora nos dois grupos, sendo esta mais evidente no 
grupo experimental, evidenciando que a associação da fonoterapia com a acupuntura é mais eficaz no tratamento da paralisia facial periférica.

Magalhães Júnior (2009) em um estudo de meta-análise de artigos publicados desde 1995, tendo como base a caracterização da paralisia facial periférica e suas relações com os distúrbios fonoaudiológicos relacionados às desordens na mobilidade orofacial. A literatura abordou a contribuição da fonoterapia na reabilitação da simetria facial. E a abordagem miofuncional orofacial contextualizou o trabalho fonoaudiológico na melhora da coordenação fonoarticulatória e no ganho do controle oral durante a mastigação e deglutição. O resultado foi satisfatório, mas há necessidade de mais publicações na abordagem miofuncional e orofacial da paralisia facial periférica.

Outro método utilizado no tratamento da paralisia fácil periférica de Bell é a ozonoterapia. García, López, Álvarez e Guerra (2008) realizaram um estudo experimental com o objetivo de avaliar o uso de ozonoterapia para reabilitação de pacientes com paralisia facial periférica de Bell. Foram utilizados 134 pacientes divididos em 2 grupos de 67 pessoas, na qual um grupo controle recebeu o tratamento convencional utilizados em otorrinolaringologia, medicina interna e reabilitação das distintas áreas de saúde para esta afecção (tais como corticoterapia, vitaminoterapia, fisioterapia e eletroestimulação) e o grupo de estudo recebeu ozonoterapia. Ao final do ciclo de tratamento do grupo de estudo, foi comprovado que 64 pacientes $(91,5 \%)$ obtiveram notáveis melhora, onde ao final do tratamento apenas 3 pacientes $(8,5 \%)$, não eliminaram todos os sintomas. Já no grupo controle, ao final do tratamento apenas 40 pacientes $(59,7 \%)$ obtiveram melhora significativa. Após este estudo conclui-se que o uso de ozonoterapia em pacientes com paralisia facial demonstrou benefícios na recuperação, reintegração dos pacientes mais rapidamente à sociedade e melhora na qualidade de vida.

\section{Tratamento Medicamentoso}

Corticosteróides orais têm sido tradicionalmente prescritos para reduzir a inflamação do nervo facial em pacientes com paralisia de Bell. Prednisona é normalmente é prescrito em um curso de 10 dias iniciando a partir de $60 \mathrm{mg}$ por dia. Maiores estudos prospectivos são necessários para estabelecer o benefício dos corticosteróides (Tiemstra e Khatkhate, 2007).

Devido ao possível papel do HSV-1 na etiologia da paralisia de Bell, os medicamentos antivirais Aciclovir (Zovirax ${ }^{\circledR}$ ) e o Valaciclovir (Valtrex ${ }^{\circledR}$ ) são estudados para determinar se existe algum benefício no tratamento. Aciclovir $400 \mathrm{mg}$ pode ser dada cinco vezes por dia durante sete dias ou Valaciclovir $1 \mathrm{~g}$ pode ser administrada três vezes por dia durante sete dias (Tiemstra e Khatkhate, 2007) (Tabela 1).

Brosens e Botargues (2008) realizaram um estudo entre 2004 a 2006, na qual foram analisados 551 pacientes com o diagnóstico de paralisia facial periférica, recrutados dentro de 72 horas do começo dos sintomas. Os pacientes divididos de acordo com terapêutica empregada, tratados apenas com prednisona, apenas com aciclovir, ambos e placebo. Após 9 meses os resultados encontrados foram similares entre os grupos, concluindo-se que a prednisona foi altamente efetiva como monoterapia e em menor medida associada ao aciclovir. Por outro lado, o aciclovir se mostrou ineficaz como tratamento único. $\mathrm{O}$ estudo demonstrou ainda que a paralisia facial periférica apresentou elevadas taxas de resolução espontânea, que foram confirmadas pela recuperação completa dos pacientes que receberam placebo, totalizando 85,2\% após os 9 meses.

Hato et al. (2007) em um estudo multicêntrico, randomizado, placebo-controlado que investigou os efeitos do valaciclovir e prednisolona em comparação com os efeitos do placebo e prednisolona no tratamento da paralisia de Bell, excluindo sinais de herpes zoster. Demonstrou que a taxa global de recuperação dos pacientes entre os tratados com valaciclovir e prednisolona $(96,5 \%)$ foi significativamente melhor do que a taxa entre os tratados com prednisolona e placebo

Revista de Saúde, Vassouras, v. 3, n. 2, p. 40-48, jul./dez., 2012 


\section{Paralisia Facial Periférica de Bell: Atualização do Tratamento}

Igor Henrique Balandino Silva, Tiago de Souza Lopes, Elisa Nocchi de Mello Motta, Juliane Lemos Araújo Deveras, Paula Corrêa Côrtes, Carla Carvalho Silva Marques, Clara Régia de Sousa Rosa, Vinicius Florentino da Silva e Ana Beatriz Calmon Nogueira da Gama Pereira

(89,7\%), sendo assim o valaciclovir associado prednisolona foi mais eficaz no tratamento de paralisia de Bell.

Tabela 1. Medicamentos para tratamento da paralisia de Bell

\begin{tabular}{|c|c|c|}
\hline Medicação & Dosagem & Reações Adversas \\
\hline $\begin{array}{l}\text { Aciclovir } \\
\text { (Zovirax®) }\end{array}$ & $\begin{array}{l}\text { Adultos: } 400 \mathrm{mg} \text { - } 5 \\
\text { vezes ao dia/7 dias } \\
\text { Crianças maiores de } 2 \\
\text { anos: } 80 \mathrm{mg} / \mathrm{kg} \text { - } 6 / 6 \\
\text { horas por } 5 \text { dias, com } \\
\text { dose máxima de } \\
3.200 \mathrm{mg} / \text { dia }\end{array}$ & $\begin{array}{l}\text { Desarranjo Gastrointestinal, cefaleia, vertigem, } \\
\text { elevação de enzimas hepáticas, anemia aplásica } \\
\text { (rara). }\end{array}$ \\
\hline $\begin{array}{l}\text { Valaciclovir } \\
\text { (Valtrex®) }\end{array}$ & $\begin{array}{l}\text { Adultos e crianças } \\
\text { acima de } 12 \text { anos: } 1 \mathrm{~g}- \\
3 \text { vezes/dia por } 7 \text { dias }\end{array}$ & $\begin{array}{l}\text { Desarranjo Gastrointestinal, cefaléia, vertigem, } \\
\text { elevação de enzimas hepáticas, anemia aplásica } \\
\text { (rara). }\end{array}$ \\
\hline $\begin{array}{l}\text { Prednisona } \\
\text { ou } \\
\text { Prednisolona }\end{array}$ & $\begin{array}{l}\text { Adultos: } 60 \mathrm{mg} / \text { dia por } \\
5 \text { dias, depois } \\
40 \mathrm{mg} / \text { dia por } 5 \text { dias } \\
\text { Crianças: } 2 \mathrm{mg} / \mathrm{kg} / \mathrm{dia} \\
\text { por } 10 \text { dias }\end{array}$ & $\begin{array}{l}\text { Cefaleia, nervosismo, edema, elevação da PA, } \\
\text { hiperglicemia. }\end{array}$ \\
\hline
\end{tabular}

*Baseado em Tiemstra, J. D., Khatkhate, N., Bell's Palsy: Diagnosis and Management.

American Family Physician 2007;76(7):997-1002.

Bauso (2006) em seu estudo de atualização evidenciou as diversas estratégias de tratamento da paralisia facial periférica. Corticosteróides e agentes antivirais isolados ou em associação, cuidados oculares, cirurgias descompressivas e terapia física são os métodos terapêuticos mais utilizados atualmente. Inúmeras pesquisas buscam comprovações de efetividade de novos métodos de tratamento (Tabela 2).

Tabela 2. Outros tratamentos emergentes

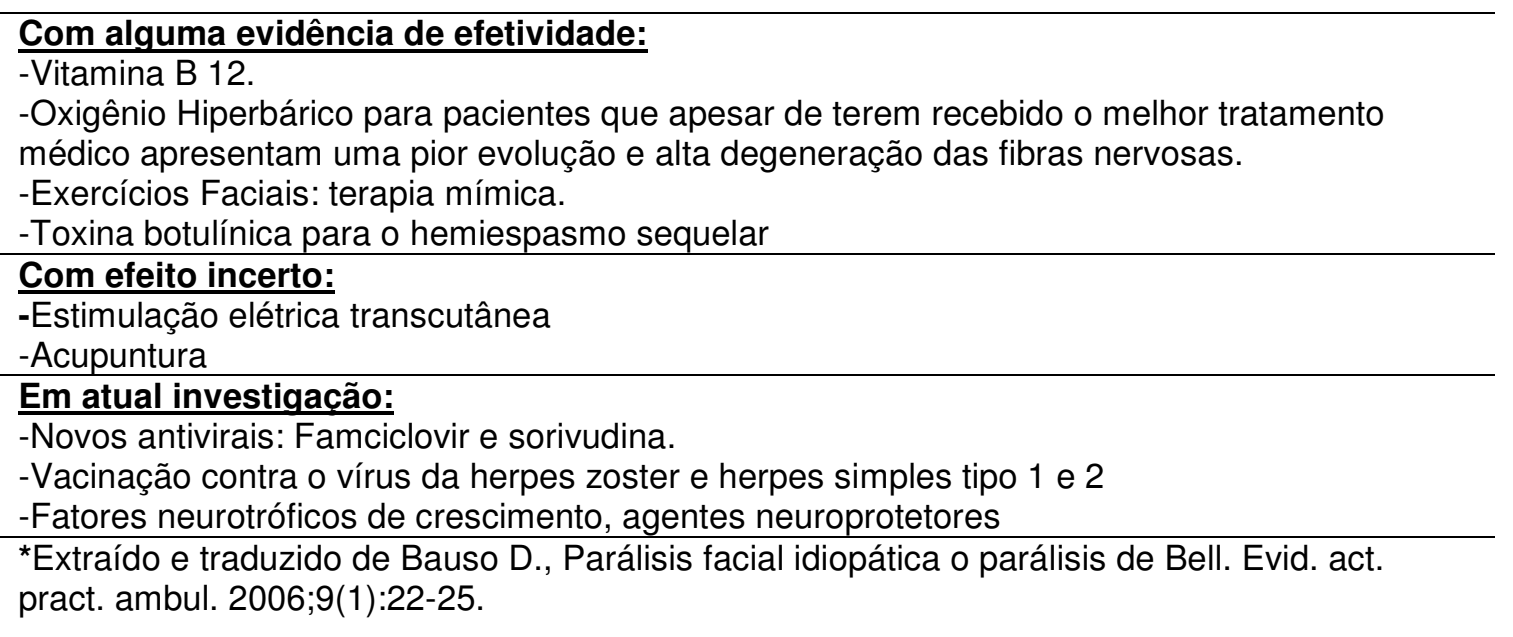

\section{Conclusão}

Há ainda uma grande divergência na literatura com relação ao tratamento e abordagem da paralisia de Bell, inúmeros estudos mostram de maneira oposta resultados com relação as suas práticas e 


\section{Paralisia Facial Periférica de Bell: Atualização do Tratamento}

Igor Henrique Balandino Silva, Tiago de Souza Lopes, Elisa Nocchi de Mello Motta, Juliane Lemos Araújo Deveras, Paula Corrêa Côrtes, Carla Carvalho Silva Marques, Clara Régia de Sousa Rosa, Vinicius Florentino da Silva e Ana Beatriz Calmon Nogueira da Gama Pereira

conclusões, dessa maneira devem-se avaliar os métodos e técnicas empregados em tais estudos, para que assim se possa determinar o tratamento mais adequado para a paralisia de Bell.

Ressaltamos ainda o grande benefício que os tratamentos combinados exercem no melhor prognóstico do paciente. E indicamos o uso de prednisolona juntamente com o valaciclovir no momento do diagnóstico da paralisia, além de adjunto ser realizada medidas que visem a reabilitação precoce e que minimizem as suas complicações.

Sendo assim, fica evidente que o tratamento da paralisia de Bell ainda necessita de mais estudos para que se possa determinar de maneira efetiva uma melhor abordagem terapêutica.

\section{Referências}

Bauso, D. (2006). Parálisis facial idiopática o parálisis de Bell. Evid. Act. pract. ambul. 9(1), 22-25.

Brosens, C., Botargues, M. (2008). ¿Son útiles los corticoides y los antivirales en el tratamiento de la parálisis facial idiopática? Evid. actual. práct. Ambul, 11(4), 124-125.

Chávez, E. P., Martínez, C. G., González, J. M. G., Rodríguez, D. E., Roldán, V. M. L., Oca, R. D. M., (2004). Guía clínica para la rehabilitación del paciente con parálisis facial periférica. Rev Med IMSS, 42(5), 425-436.

Diego, J. I., Prim, M. P., Gavilán J. (2001). Etiopatogenia de la parálisis facial periférica idiopática o de Bell. Rev Neurol, 32(11), 1055-1059.

Falavigna, A., Teles, A. R., Giustina, A. D., Kleber, F. D. (2008). Paralisia de Bell: fisiopatologia e tratamento. Scientia Medica, 18(4), 177-183.

Finsterer, J. (2008). Management of peripheral facial nerve palsy. Eur Arch Otorhinolaryngol, 265, 743-752.

García, A. A., López, M. P., Álvarez, J. S., Guerra, I. C. (2008). Ozonoterapia como tratamiento rehabilitador de la parálisis facial periférica tipo Bell. Arch. Méd. Camaguey, 12(5).

Goulart, F., Vasconcelos, K. S. S., Souza, M. R. V., Pontes, P. B. (2002). A utilização do biofeedback no tratamento fisioterápico da paralisia facial periférica. Acta Fisiátrica, 9(3), 134140.

Hato, N., Yamada, H., Kohno, H., Matsumoto, S., Honda, N., Gyo, K. (2007). Valacyclovir and Prednisolone Treatment for Bell's Palsy: A Multicenter, Randomized, Placebo-Controlled Study. Otology \& Neurotology, 28, 408-413.

Júnior, H. V. M. (2009). Fonoterapia na Paralisia Facial Periférica: Uma Abordagem Miofuncional Orofacial. RBPS, 22(4), 259-263.

Lazarini, P. R., Vianna, M. F., Alcantara, M. P. A., Scalia, R. A., Filho, H. H. C. (2006). Pesquisa do vírus Herpes simples na saliva de pacientes com paralisia facial periférica de Bell. Rev. Bras. Otorrinolaringol, 72(1), 7-11.

Resende, L. A. L., Weber, S. (2008). Peripheral facial palsy in the past. Arq Neuropsiquiatr, 66(3B), 765-769.

Rosa, M. C. P.; Moreira, A. F. M.; Araújo, L. B.; Júnior, L. C. M., Motta, A. R. (2010). Comparação dos resultados da fonoterapia e da fonoterapia associada à acupuntura na paralisia facial periférica. Rev. CEFAC, 12(4), 579-588.

Santos, M. A. O., Filho, H. H. C., Viana, M. F., Almeida, A. G. P., Lazarini, P. R. (2010). Varicela zoster vírus in Bell's palsy: a prospective study. Braz J Otorhinolaryngol, 76(3), 370-3.

Revista de Saúde, Vassouras, v. 3, n. 2, p. 40-48, jul./dez., 2012 


\section{Paralisia Facial Periférica de Bell: Atualização do Tratamento}

Igor Henrique Balandino Silva, Tiago de Souza Lopes, Elisa Nocchi de Mello Motta, Juliane Lemos Araújo Deveras, Paula Corrêa Côrtes, Carla

Carvalho Silva Marques, Clara Régia de Sousa Rosa, Vinicius Florentino da Silva e Ana Beatriz Calmon Nogueira da Gama Pereira

Soares, A. C. C., Silva, L. R., Bertolini, S. M. M. G. (2002). Atuação da fisioterapia na paralisia facial periférica: relato de caso. Arq. Ciênc. Saúde Unipar, 6(3), 171-176.

Tessitore, A., Paschoal, J. R., Pfeilsticker, L. N. (2009). Avaliação de um protocol da reabilitação orofacial na paralisia facial periférica. Rev CEFAC, 11(3), 432-440.

Teixeira, L. J., Soares, B. G., Vieira, V. P., Prado, G. F. (2008). Physical therapy for Bell s palsy (idiopathic facial paralysis). Cochrane database of syst. Rev., 16(3), 6283.

Tiemstra, J. D., Khatkhate, N. (2007). Bell's Palsy: Diagnosis and Management. American Family Physician, 76(7), 997-1002.

Valença, M. M., Valença, L. P. A. A., Lima, M. C. M. (2001). Paralisia Facial Periférica Idiopática de Bell: A propósito de 180 pacientes. Arq. Neuropsiquiatr, 59(3-B), 733-739. 